\title{
Dependence of Shortening Heat on Sarcomere Length in Frog Muscle and Fiber Bundles
}

\author{
Kaoru Kometani and Kazuhiro Yamada \\ Department of Physiology, Medical College of Oita, \\ Oita, 879-56 Japan
}

\begin{abstract}
The relation between shortening heat and sarcomere length was studied using fiber bundles from frog semitendinosus muscles as well as using whole muscles. The initial sarcomere length was varied between 2.0 and $3.66 \mu \mathrm{m}$. Shortening heat was estimated as the excess heat produced after a rapid isovelocity release in a $3 \mathrm{sec}$ tetanus at $0^{\circ} \mathrm{C}$. The isometric control heat was measured in the same tetanus, before and after the period of shortening. The unstimulated whole muscles showed a large thermoelastic absorption of heat when released at sarcomere lengths longer than $2.5 \mu \mathrm{m}$, and the apparent shortening heat was negative at very long sarcomere lengths. The apparent shortening heat was corrected by subtracting the thermoelastic heat absorption by assuming that the thermoelastic effect was also present in releases of active muscles. The corrected shortening heat decreased linearly with increasing sarcomere length in the range $2.29-3.66 \mu \mathrm{m}$, intersecting the length axis at $3.73 \pm 0.21 \mu \mathrm{m}$. The thermoelastic heat absorption at long sarcomere lengths was substantially reduced in fiber bundles, suggesting that the parallel elasticity responsible for the thermoelastic effect is mainly present outside muscle cells. The corrected shortening heat in fiber bundles also decreased linearly with increasing sarcomere length, intersecting the length axis at $3.84 \pm 0.25 \mu \mathrm{m}$. Thus the results on fiber bundles, also based on correction but the extent of which is substantially smaller than in whole muscles, are in agreement with the results on whole muscles. The results are interpreted to mean that shortening heat is produced by the interaction of thick and thin filaments in contracting muscle.
\end{abstract}

Key Words: muscle, shortening heat, sarcomere length, thermoelasticity.

When an active muscle is allowed to shorten, heat is produced at a greater rate than in isometric contraction. The heat produced in shortening muscles in

Received for publication June 28,1983

米谷快男児, 山田和廣 
excess of that in isometrically contracting ones is known as the shortening heat (HILL, 1938). Shortening heat presumably represents extra chemical reactions caused by the greater rate of cross-bridge turnover during shortening. It has been shown, however, that shortening heat in rapidly shortening muscles is not accompanied by simultaneous high energy phosphate splitting (KUSHMERICK et al., 1969; RALl et al., 1976; Homsher et al., 1981). Homsher et al. (1981) have shown that the unexplained enthalpy during rapid shortening is reversed in a post-shortening period, at the same time being accompanied by enthalpy production in excess of that in muscles without shortening. These results suggest that the shortening heat originates in the shift in the population of cross-bridge states during rapid shortening, and the transition is reversed afterwards, being coupled to ATP splitting. Cross-bridge mechanisms to account for the above findings have been proposed by several authors (CURTIN and Woledge, 1978; HoMSHER and Kean, 1978; Kodama and Yamada, 1979).

According to the independent force-generator theory (GoRDON et al., 1966), shortening heat in rapidly shortening muscles is expected to be directly proportional to the extent of overlap between thick and thin filaments in each half-sarcomere. The relation between shortening heat and sarcomere length was first studied by LEBACQ (1972) using frog sartorius muscles. He briefly reported that as sarcomere length was increased shortening heat declined more steeply than could be predicted from the extent of filament overlap. He suggested that the discrepancy could be explained by large changes in resting tension caused by shortening at long muscle lengths. IRVING et al. (1980) and YAMADA et al. (1981) studied the same problem using frog semitendinosus muscles, which is known to permit extensive stretching without injury (HoMsher et al., 1972). Both authors briefly reported that the unstimulated muscles absorbed heat thermoelastically when allowed to shorten at sarcomere lengths longer than $3 \mu \mathrm{m}$, and corrected the apparent shortening heat for this heat absorption. IRVING et al. (1980) found that the dependence of the corrected shortening heat on sarcomere length was similar to that of the isometric tension, whereas YAMADA et al. (1981) failed to find the linear dependence. The discrepancy between the two was probably caused by the large correction involved, especially when the number of measurements was limited as in YAMADA et al. (1981). IRVING and Woledge (1981) have also reported that, in frog sartorius muscles, the shortening heat after small correction for the heat absorption in unstimulated muscles showed the similar dependence to the isometric tension in the sarcomere length range between 2 and $2.6 \mu \mathrm{m}$.

Here we report the results of our reinvestigation of the same problem. The results showed that shortening heat, after allowance was made for the thermoelastic heat absorption on releasing resting pre-stretched muscles, linearly depended on sarcomere length as isometric tension did in the sarcomere length range between 2.2 and $3.66 \mu \mathrm{m}$. We also confirmed the above relation using fiber bundles, which produced substantially less thermoelastic heat absorption than whole muscles. 


\section{METHODS}

Muscles and the preparation of fiber bundles. Ventral heads of the semitendinosus muscles from Rana japonica were used, attachment of the tendons on the pelvic bone being preserved. Experiments were performed on fiber bundles as well as on whole muscles. In most experiments, one of a pair of muscles was used to obtain a bundle of fibers and the other was used for whole muscle experiments. Bundles of fibers were dissected under stereoscopic magnification using micro-scissors. The fiber bundles were about 1 to $1.2 \mathrm{~mm}$ in diameter and were about one third to one half of whole muscles in cross-sectional area. Ringer solution contained (mM): $\mathrm{NaCl}, 115 ; \mathrm{KCl}, 2.5 ; \mathrm{CaCl}_{2}, 1.8 ; \mathrm{NaH}_{2} \mathrm{PO}_{4}, 0.68 ; \mathrm{Na}_{2} \mathrm{HPO}_{4}, 1.3$ at $\mathrm{pH}$ 7.0 at $0^{\circ} \mathrm{C}$ and was bubbled with $\mathrm{O}_{2}$. Muscles were stimulated directly via platinum electrodes located near the top and the bottom ends of the muscle, using square pulses of $2 \mathrm{msec}$ duration at about $10 \mathrm{~Hz}$ at $0^{\circ} \mathrm{C}$.

Control of muscle length. The muscles were connected via force and length transducers to a Ling 200 series vibration generator. The force transducer was made of an aluminium block to which strain gauges had been bonded. The photoelectric length transducer was of the type described by JEWELL et al. (1967) utilizing a strip of optically wedged photographic film. Muscle length was made to follow a control signal by a feed-back network driving the vibration generator. The effective stiffness of transducers and feed-back system was $6.6 \mathrm{kN} / \mathrm{m}$.

Shortening heat measurements. Two thermopiles (K1 and K3) of the electroplated type (RICCHIUTI and MommaERTS, 1965) were used for heat measurements. For the earlier part of the experiments thermopile $\mathrm{K} 1$ was used. It has overall length of $25 \mathrm{~mm}$, and a $9 \mathrm{~mm}$ region, with a sensitivity of $3.0 \mathrm{mV} / \mathrm{deg}$, was used for heat measurements. For the later part of the experiments a $7 \mathrm{~mm}$ region of thermopile $\mathrm{K} 3$ with a sensitivity of $1.99 \mathrm{mV} / \mathrm{deg}$, whose overall length is $11 \mathrm{~mm}$, was used. The equivalent half-thickness (HILL, 1965) of both of these thermopiles was $14 \mu \mathrm{m}$ as estimated from heat capacities of the materials constituting them. The thermopile output was amplified by an Ancom 15C-3a chopper amplifier, low-pass filtered (cut-off frequency, $200 \mathrm{~Hz}$ ) and recorded on a Nicolet 2090 digital oscilloscope. Calibration of the heat produced by muscle was made by the method of HiLl and Woledge (1962). Heat loss from the muscle was exponential with rate constants between 0.07 and $0.11 \mathrm{sec}^{-1}$ for bundles and between 0.02 and $0.06 \mathrm{sec}^{-1}$ for whole muscles. The rate constants were consistently larger at longer sarcomere lengths. Experimental records were corrected for heat loss by the method of HiLL (1965). A correction for the lag in heat conduction from muscles to thermopiles was not made. In view of the small equivalent halfthickness of the thermoplies the extent of this correction should be small. Considering the method used to estimate shortening heat, this would not affect the present results in any appreciable way.

Muscles were mounted vertically on one side of the thermopile and connected to the force and length transducers. At least $1 \mathrm{hr}$ was allowed for thermal 
equilibration in aerated Ringer solution at $0^{\circ} \mathrm{C}$. After Ringer solution was drained the muscles were tetanically stimulated for $3 \mathrm{sec}$. At $1.5 \mathrm{sec}$ after the onset of stimulation, muscles were allowed to shorten by 0.2 to $0.3 \mu \mathrm{m}$ per sarcomere in 0.1 to $0.12 \mathrm{sec}$ at a constant velocity, nearly equal to the maximum shortening velocity for each muscle. Shortening heat was defined as described by IRVING et al. (1979): the heat produced in $0.4 \mathrm{sec}$ after the onset of shortening in excess of the heat of isometric control calculated as a weighted average of the isometric heats at long and short lengths, corresponding to the periods before and after the controlled shortening. After an observation of two contractions, isometric and isometric with shortening, muscles were immersed in Ringer solution, changed their length, and stood for about $15 \mathrm{~min}$ before a subsequent observation. Muscles were stretched to a length corresponding to the sarcomere length of $3 \mu \mathrm{m}$ in about $0.15 \mu \mathrm{m} /$ sarcomere steps. Muscles were then released, and measurements were repeated to check the reversibility. This time, muscles were stretched beyond the length corresponding to the sarcomere length of $3 \mu \mathrm{m}$.

Sarcomere length measurements. Sarcomere length was measured in the unstimulated muscle by diffraction with $25 \mathrm{~mW}$ He-Ne laser beam of $1.2 \mathrm{~mm}$ diameter. Measurements were made with muscles on the thermopile each time muscle lengths were changed. Mean sarcomere length was calculated from the separation of the center of the first order diffraction line from that of the zero-order line on a vertical screen. The larger proportion of the optical path $(11 \mathrm{~cm})$ from muscle to the screen was through water phase. This was taken into account in calculating the sarcomere length from the diffracted beam. The calculation was checked using a diffraction grating of $10 \mu \mathrm{m}$ spacing (objective micrometer, Nikon). Measurements were made at two points along the muscle, one point at the central region and the other near the tibial tendon, and the average of the two was taken as the sarcomere length of the muscle. The length of muscle fiber which corresponded to the sarcomere length of $2.2 \mu \mathrm{m}$ was taken as the standard length $\left(l_{0}\right)$ of muscle fibers. The value of $l_{0}$ was estimated from the relation between changes in muscle length and changes in sarcomere length. The cross-sectional area of muscle was estimated by dividing the blotted weight of muscle by $l_{0}$.

\section{RESULTS}

Heat production associated with shortening in pre-stretched muscles

Figures 1, 2, and 3 show records of muscle length (trace 1), tension (2 and $\left.2^{\prime}\right)$ and heat production $\left(3\right.$ and $\left.3^{\prime}\right)$ in $3 \mathrm{sec}$ tetani. Figure 1 shows the effect of shortening $(0.25 \mu \mathrm{m} /$ sarcomere $)$ from the initial sarcomere length of $2.29 \mu \mathrm{m}$ in a whole muscle. The muscle was tetanically stimulated for $3 \mathrm{sec}$ and was released at $1.5 \mathrm{sec}$ after the onset of stimulation at a velocity of $1.0 l_{0} / \mathrm{sec}$ corresponding to $2.22(\mu \mathrm{m} / \mathrm{sarcomere}) / \mathrm{sec}$, so that the active tension fell to a value close to the resting tension (2). Heat production for $1.5 \mathrm{sec}$ after the onset of stimulation 

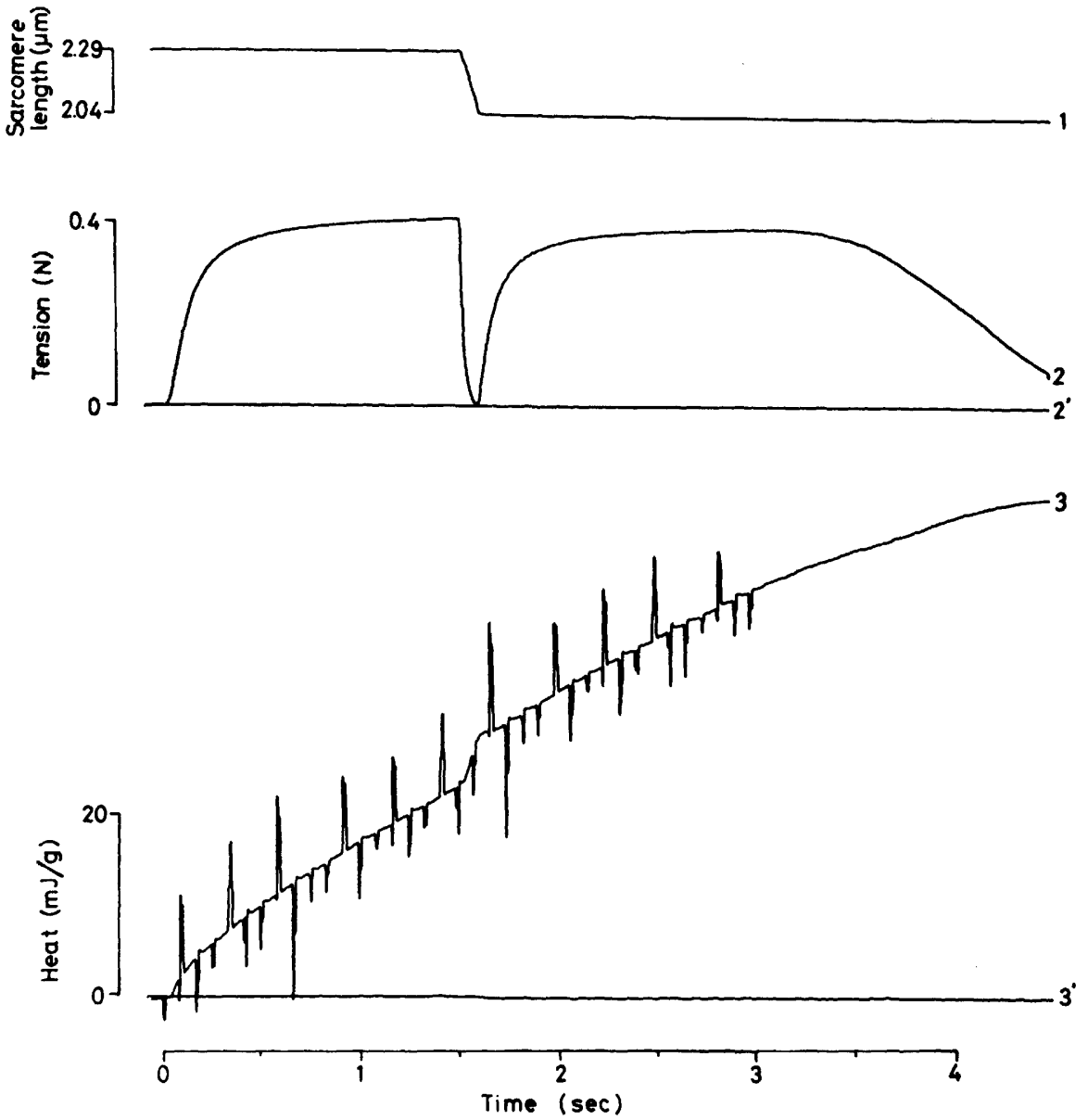

Fig. 1. Muscle length, tension, and heat production in a $3 \mathrm{sec}$ tetanus of a frog semitendinosus muscle at $0^{\circ} \mathrm{C}$. The muscle contracted isometrically for $1.5 \mathrm{sec}$ at long length, then was allowed to shorten from 2.29 to $2.04 \mu \mathrm{m} /$ sarcomere at a constant velocity of 2.22 $(\mu \mathrm{m} / \mathrm{sarcomere}) / \mathrm{sec}$. Traces 1 (length), 2 (tension), and 3 (heat production) are records for active muscle. When the same length change was applied to unstimulated muscle, traces 2 ' (tension) and $3^{\prime}$ (heat production) were obtained. The spikes on the heat record are artefacts due to stimuli. Blotted weight of muscle, $22.4 \mathrm{mg}$; standard length of muscle fiber, $12.3 \mathrm{~mm}$.

represents an isometric maintenance heat (3). Heat is seen to be produced at a higher rate associated with the release. Shortening heat was measured in a single tetanus by estimating the isometric control heat production in the same tetanus before and after a period of shortening (IRVING et al., 1979; see METHODs). When the same release was applied in unstimulated muscle, the changes in tension $\left(2^{\prime}\right)$ and heat $\left(3^{\prime}\right)$ were both undetectably small.

Vol. 33, No. 6, 1983 


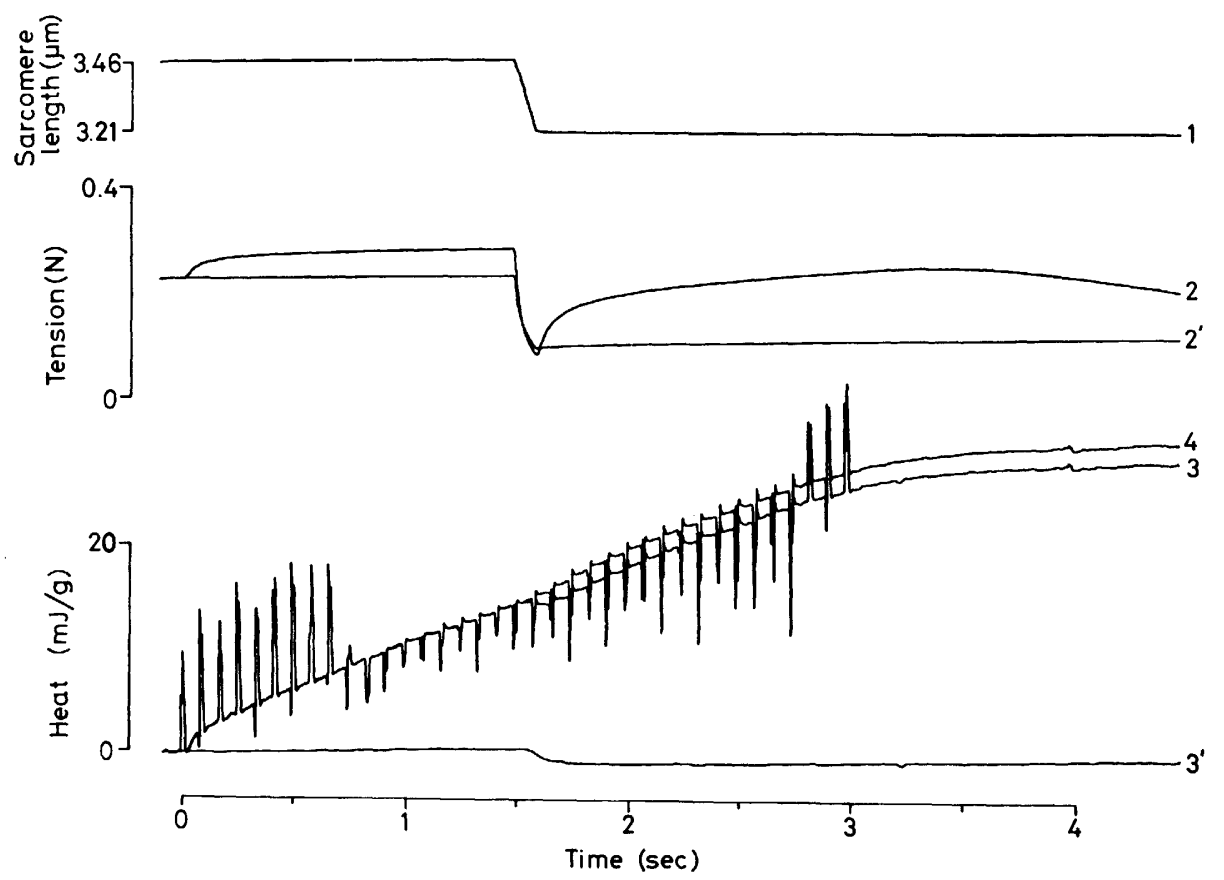

Fig. 2. As Fig. 1 except that the isovelocity shortening was from 3.46 to $3.21 \mu \mathrm{m} /$ sarcomere at $2.22(\mu \mathrm{m} / \mathrm{sarcomere}) / \mathrm{sec}$. Trace 4 shows the result where trace $3^{\prime}$ was subtracted from trace 3.

Figure 2 shows the effect of similar shortening from the initial sarcomere length of $3.46 \mu \mathrm{m}$ in a pre-stretched whole muscle. The pre-stretched muscle showed a large resting tension, so that when released without stimulation the tension was reduced to a lower level (trace $2^{\prime}$ ). On stimulation, a small tension was produced corresponding to the extent of remaining overlap between the filaments (2). When released, the active tension fell to a low level (2), which almost coincided with the level attained by the pre-stretched muscles when released without stimulation $\left(2^{\prime}\right)$. Heat is seen to have been aborbed when active muscles were released (3), and the absorption of heat is also seen in the unstimulated muscles $\left(3^{\prime}\right)$. We may reasonably assume that the absorption of heat on release occurred in active muscles as well as in unstimulated muscles. Trace 4 shows the results where the heat record of unstimulated muscle ( $\left.3^{\prime}\right)$ was subtracted from the active muscle (3). Shortening heat is seen to have been reduced to a very small value (4).

Figure 3 shows the results of similar experiments to those shown in Fig. 2, using a fiber bundle preparation. The results are essentially similar to those in Fig. 2 except that the absorption of heat on releasing the unstimulated muscle $\left(3^{\prime}\right)$ is substantially less than that in whole muscle (trace $3^{\prime}$ in Fig. 2). 

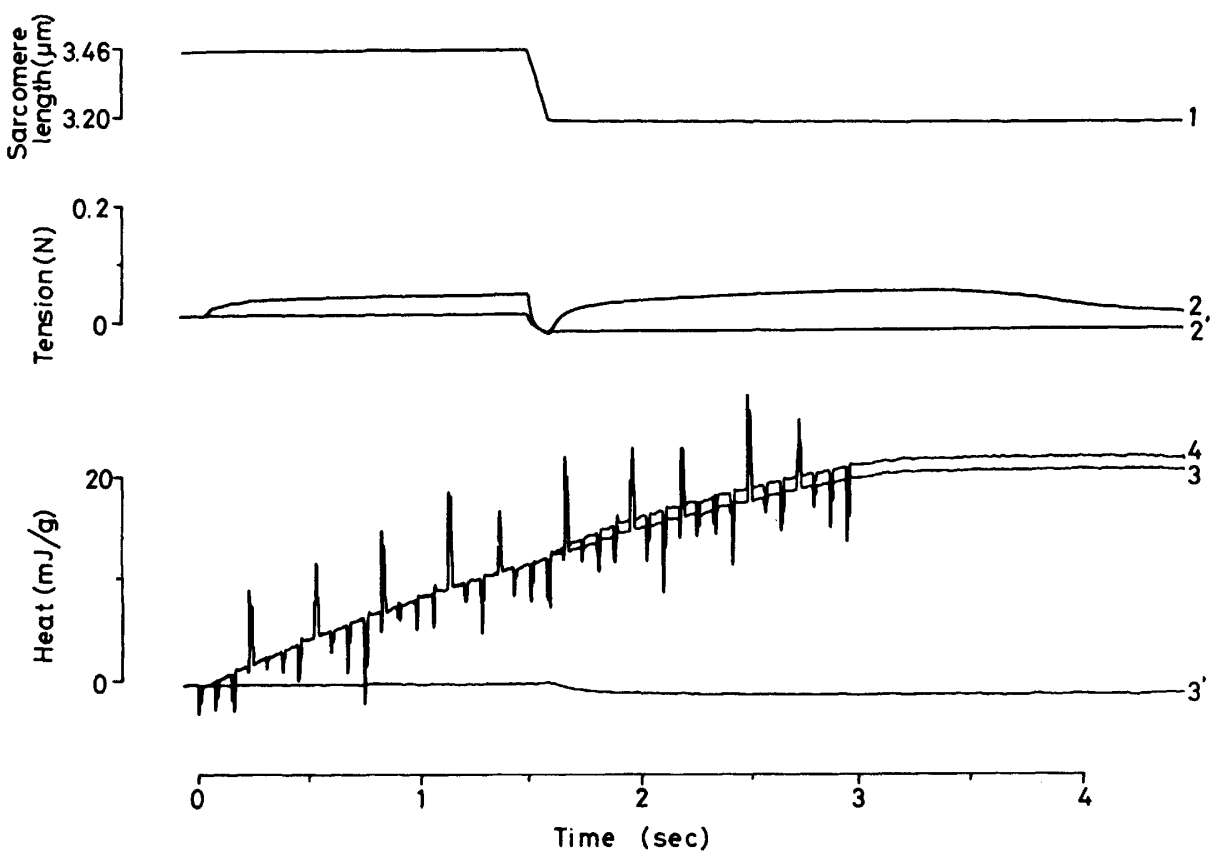

Fig. 3. As Fig. 2 except that a fiber bundle was used. Isovelocity shortening of $2.31(\mu \mathrm{m} /$ sarcomere)/sec; blotted weight of fiber bundle, $8 \mathrm{mg}$; standard length of muscle fiber, $9.86 \mathrm{~mm}$.

The relation between isometric tension and sarcomere length

Figure 4-b shows the dependence of isometric tension on sarcomere length in whole muscles. Isometric tension was measured immediately before release and normalized for each muscle by the value $\left(P_{0}\right)$ at the sarcomere length of $2.29 \mu \mathrm{m}$. The value of $P_{0}$ was $225 \pm 59 \mathrm{kN} / \mathrm{m}^{2}$ (mean \pm S.E. of mean, $n=7$ ). In whole muscles, isometric tension declined linearly as sarcomere length was increased from 2.29 to $3.66 \mu \mathrm{m}$. Figure 6-b shows the dependence of isometric tension on sarcomere length in fiber bundles. The value of $P_{0}$ in fiber bundles was $164 \pm$ $34 \mathrm{kN} / \mathrm{m}^{2}$ (mean $\pm \mathrm{S}$.E. of mean, $n=11$ ). In fiber bundles, isometric tension also declined linearly as sarcomere length was increased. Linear regression of isometric tension in each muscle with sarcomere length indicates that isometric tension would be reduced to zero at $3.73 \pm 0.08 \mu$ m (mean \pm S.E. of mean, $n=7$ ) in whole muscles and $3.84 \pm 0.17 \mu$ m (mean \pm S.E. of mean, $n=10$ ) in fiber bundles.

When muscles are stimulated at long sarcomere lengths isometric tension creeps up slowly. This slow rise in tension is usually attributed to a progressive increase in the dispersion of sarcomere length along muscle fibers (GORDON et al., 1966). Isometric tension in Figs. 4 and 6 was measured at $1.5 \mathrm{sec}$ after the start of stimulation. Hence the sarcomere length at which isometric tension would 
be reduced to zero (Figs. 4 and 6) is expected to be somewhat longer than that obtained by GoRDON et al. (1966).

The relation between shortening heat and sarcomere length in whole muscles

Figure 4-a shows the relation between shortening heat and sarcomere length in whole muscles. Because shortening heat was measured by allowing muscles to shorten by 0.2 to $0.3 \mu \mathrm{m}$ per sarcomere, shortening heat was converted to the
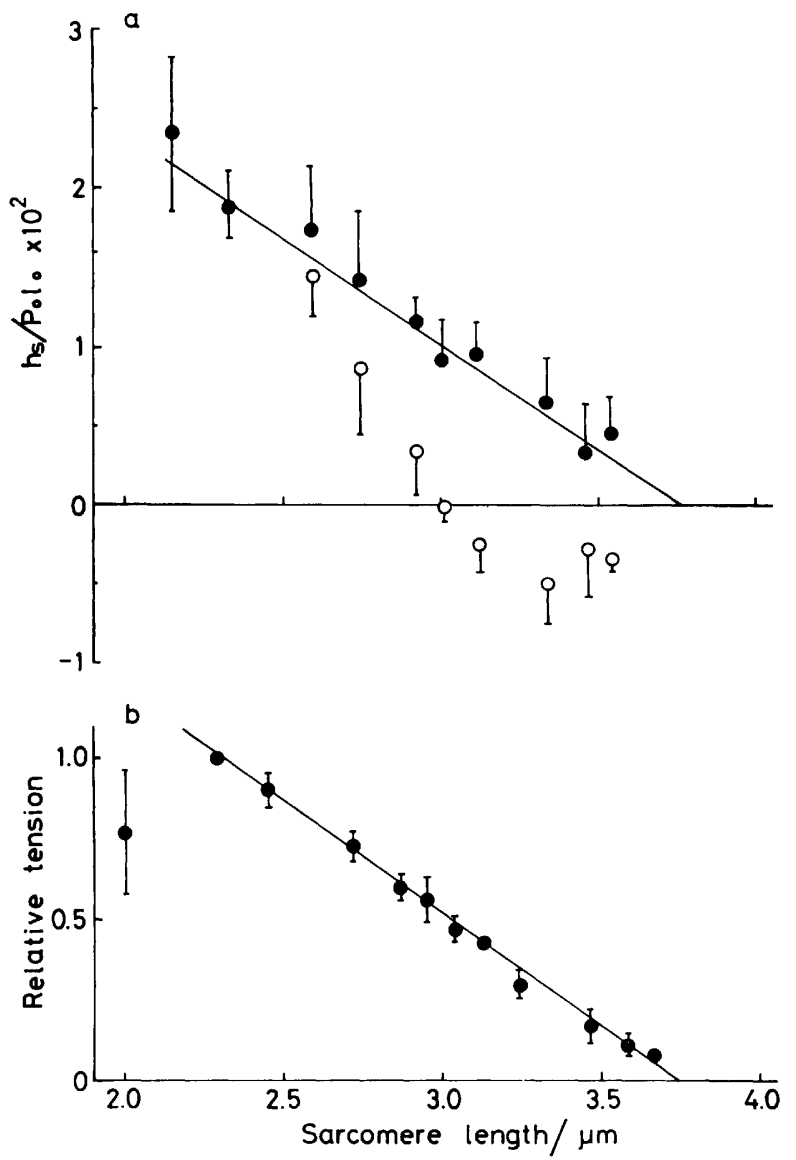

Fig. 4. a. Relation between shortening heat and sarcomere length in whole muscles. Open circles, apparent shortening heat; closed circles, shortening heat corrected for the thermoelastic heat absorption. Each point (mean \pm S.E. of mean, $n=4-15$ ) is plotted against the midpoint of $0.25 \mu \mathrm{m}$ shortening. The straight line was obtained by linear regression of the mean value of the corrected shortening heat at each length. b. Relation between isometric tension and sarcomere length in whole muscles. Tension was normalized for each muscle by the value at the sarcomere length of $2.29 \mu \mathrm{m}$. The straight line was obtained by linear regression of the mean value at each length. 
value corresponding to $0.25 \mu \mathrm{m}$ shortening per sarcomere to compare results obtained from different experiments on different muscles. It was assumed that shortening heat is linearly related to the extent of shortening in the range between 0.2 and $0.3 \mu \mathrm{m}$ per sarcomere (IRVING and Woledge, 1981). Shortening heat produced per unit weight $(\mathrm{g})$ of muscle was normalized by $\boldsymbol{P}_{0}$. In Fig. 4-a the dimension-less numbers thus obtained were plotted against the midpoint of the range of sarcomere length over which the shortening took place. Shortening heat may also be expressed per unit extent of shortening, normalized by $P_{0}$ in each muscle $\left(a / P_{0} ;\right.$ Hill, 1964). The value of $a / P_{0}$ in the shortening from $2.29 \mu \mathrm{m}$ was $0.190 \pm 0.033$ (mean \pm S.E. of mean, $n=11$ ), in agreement with previously reported values (HILL, 1964; HoMSHER and RALL, 1973).

Open circles in Fig. 4-a show the apparent shortening heat determined as described in METHODS. The apparent shortening heat declined as sarcomere length was increased and became negative at sarcomere lengths longer than 3.0 $\mu \mathrm{m}$. When muscles were released at long sarcomere lengths without stimulation, they absorbed heat thermoelastically (IRvING et al., 1980; YAMADA et al., 1981). This absorption of heat seemed to be present in active shortening because the apparent shortening heat was negative at very long sarcomere lengths as described above. This absorption of heat on releasing muscles was measured separately just before or after the measurements of shortening heat. Then this was used to correct the shortening heat, assuming that the same thermoelasticity holds for active as well as for unstimulated muscles. Closed circles in Fig. 4-a show the corrected shortening heat. The corrected shortening heat, like isometric tension (Fig. 4-b), was linearly decreased as sarcomere length was increased. Linear regression of the corrrected shortening heat with sarcomere length indicated that shortening heat would be reduced to zero at the sarcomere length of $3.73 \pm$ $0.21 \mu \mathrm{m}$ (mean \pm S.E. of mean, $n=9$ ).

Thermoelastic heat absorption by releasing muscles without stimulation at long sarcomere lengths

Muscles absorbed heat thermoelastically when released at long sarcomere lengths without stimulation. This absorption of heat on releasing muscles was measured separately just before or after the measurements of shortening heat. In Fig. 5 the thermoelastic heat/tension ratio, $\triangle Q / \Delta P l_{0}$, was plotted against the initial sarcomere length, where $\Delta Q$ is the amount of heat absorbed, $\Delta P$ the change of tension on releasing and $l_{0}$ the standard length of fibers. Closed circles show the relation between the thermoelastic heat absorption and sarcomere length in whole muscles. The thermoelastic heat absorption started to appear at the sarcomere length just above $2.4 \mu \mathrm{m}$, attained its maximum at $2.92 \mu \mathrm{m}$ and then became smaller again at greater sarcomere lengths. This is caused partly by larger $\triangle P$ at longer sarcomere and partly by smaller amount of heat absorbed on release at very long sarcomeres.

Vol. 33, No. 6, 1983 


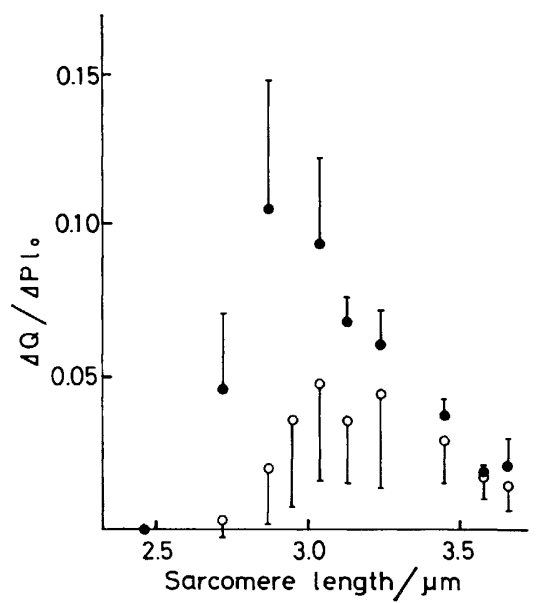

Fig. 5. Relation between the thermoelastic heat absorption and sarcomere length in whole muscles $(0)$ and in fiber bundles $(O)$. Each point represents mean \pm S.E. of mean $(n=$ 3-14) at each length.

Since thermoelastic heat absorption appears only at greater sarcomere lengths, the thermoelasticity should be caused by the parallel elastic component in muscles. In whole muscles parallel elastic component is composed of the structure inside muscle cells as well as of connective tissues existing outside muscle cells. In order to study the contribution of connective tissues fiber bundles were prepared and the thermoelastic heat absorption at various sarcomere lengths was measured. Open circles in Fig. 5 show the relation between the thermoelastic heat absorption and sarcomere length in fiber bundles. Thermoelastic heat absorption is substantially smaller at the sarcomere length range between 2.71 and $3.23 \mu \mathrm{m}$ in fiber bundles than in whole muscle. This result shows that the structures that caused the thermoelasticity mainly exist outside muscle cells.

\section{The relation between shortening heat and sarcomere length in fiber bundles}

Figure 6-a shows the relation between shortening heat and sarcomere length in fiber bundles. Shortening heat produced per unit weight (g) of muscle normalized by $P_{0}$ per unit cross-sectional area showed greater variations in fiber bundles than in whole muscles. This was most probably caused by a larger error being introduced in determining the muscle weight in fiber bundles than in whole muscles. Therefore, shortening heat was further normalized for each fiber bundle preparation by the value obtained in the shortening from $2.29 \mu \mathrm{m}$. Figure 6-a shows the normalized shortening heat at each sarcomere length (mean \pm S.E. of mean, $n=3-11$ ). Open circles show the apparent shortening heat and closed circles the shortening heat corrected for thermoelastic absorption of heat. The straight line was obtained by linear regression of the mean value of the corrected 

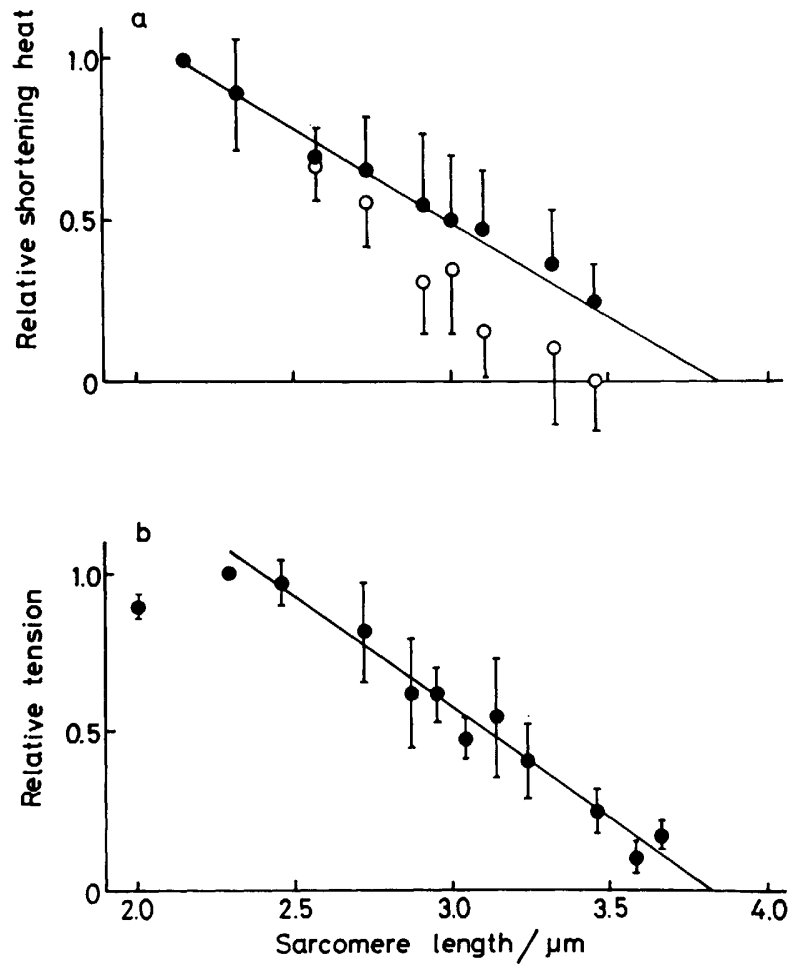

Fig. 6. a. Relation between shortening heat and sarcomere length in fiber bundles. Shortening heat was normalized for each muscle by the value for the shortening from $2.29 \mu \mathrm{m}$. Open circles, apparent shortening heat; closed circles, the corrected shortening heat. Each point (mean \pm S.E. of mean, $n=3-11$ ) is plotted against the midpoint of $0.25 \mu \mathrm{m}$ shortening. The straight line was obtained by linear regression of the mean value of the corrected shortening heat at each length. b. Relation between isometric tension and sarcomere length. Tension was normalized for each muscle by the value at sarcomere length of $2.29 \mu \mathrm{m}$. The straight line was obtained by linear regression of the mean value at each length.

shortening heat in the sarcomere length range of over $2 \mu \mathrm{m}$. Linear regression indicated that the shortening heat would be reduced to zero at sarcomere length of $3.84 \pm 0.25 \mu \mathrm{m}$ (mean \pm S.E. of mean, $n=8$ ). The value of $a / P_{0}$ was $0.136 \pm$ 0.012 (mean \pm S.E. of mean, $n=11$ ) in the shortening from $2.29 \mu \mathrm{m}$, which is somewhat lower than previously reported values in whole muscles (HILL, 1964; HOMSHER and RALL, 1973).

\section{DISCUSSION}

When an active muscle is allowed to shorten, the rate of heat production is greater than it is in an isometric contraction: this excess is defined as shortening 
heat (HILL, 1938). Near slack length, the above definition of shortening heat leads to a determination of shortening heat as the heat produced in a contraction with shortening in excess of the heat in isometric contraction (IRVING et al., 1979). At greater initial length, however, the process of shortening heat determination becomes complicated because resting tension becomes significant, and a substantial amount of heat is absorbed on releasing unstimulated muscles. In this study we have reported the linear dependence of shortening heat on the extent of filament overlap, by examining the variation of shortening heat with sarcomere length, under the assumption that the thermoelastic effect was present in the same extent in both active and unstimulated muscles.

As Fig. 4-a shows, under this assumption, shortening heat in whole muscles has a similar dependence on sarcomere length as isometric tension does. The regression analysis of shortening heat against sarcomere length showed that the intercept was at the sarcomere length exactly corresponding to the isometric tensionsarcomere length relation. However, the above results were based on very large correction, the extent of which can be seen as the difference between the filled and the open circles.

Because the large absorption of heat on releasing unstimulated muscles was observed only at long muscle lengths, the thermoelasticity should be caused by the parallel elasticity of muscle. The elasticity responsible for the resting tension in pre-stretched, unstimulated muscles seemed to be present, almost unaffected, in active muscles in parallel with contractile component. This is because on rapid release tension in active muscles dropped to almost the same level as in unstimulated muscles as seen in traces 2 and $2^{\prime}$ in Fig. 2. This seems to support the above assumption that the thermoelastic heat was present in the same extent in both active and unstimulated muscles. The elasticity responsible for the resting tension, however, does not necessarily coincide with the amount of thermoelastic heat absorption. This is indicated in the results in Fig. 5, which show that the relation between thermoelasticity and tension in unstimulated muscles is non-linear: the thermoelastic heat/tension ratio is maximum at the sarcomere length of about $2.8 \mu \mathrm{m}$ in whole muscles. It is desirable, therefore, to use a preparation which has a less parallel elasticity.

It was found (Fig. 5) that the extent of the thermoelastic heat absorption was substantially less in fiber bundles than in whole muscles. Moreover, the sarcomere length at which the heat absorption started to appear in fiber bundles was shifted to lengths longer by about $0.3 \mu \mathrm{m} /$ sarcomere than in whole muscles. These findings indicated that the relative amount of the structures with rubber-like elasticity is less in fiber bundles than in whole muscles. These also indicated that the elastic structure is mostly present outside muscle cells. It is known that elasticity of fibrous proteins such as keratin is rubber-like (MEYER and HASELBACH, 1949). The shortening heat in fiber bundles was also corrected for the thermoelastic heat absorption, which was much smaller than in whole muscles. This 
correction can be seen as the difference between the filled and the open circles in Fig. 6-a. The corrected shortening heat also linearly declined with increasing sarcomere length, as isometric tension did, the intercept on sarcomere length axis almost corresponding to the isometric tension-sarcomere length relation.

Thus the sarcomere length dependence of shortening heat obtained in fiber bundles agreed with that in whole muscles. These results support the validity of the correction or the assumption involved in shortening heat measurements at long sarcomere lengths in both whole muscles and fiber bundles, i.e., the same thermoelasticity holds for active as well as for unstimulated muscles. The linear dependence of shortening heat on the extent of filament overlap is in agreement with the idea that shortening heat is produced by the interaction between thick and thin filaments, and also with the idea that force-generators or cross-bridges are distributed throughout each overlap zone (independent force-generators; HuXLEY, 1957, 1980).

The present study indicated that shortening heat represents the property of the cross-bridges. It has been shown, however, that the shortening heat of rapidly shortening muscles is not accompanied by high energy phosphate splitting (KUSHMERICK et al., 1969; RAll et al., 1976; HoMSHER et al., 1981). Thus shortening heat cannot originate simply from a higher turnover rate of cross-bridges. Rather the origin of shortening heat should be sought in the shift in the population of cross-bridge states during rapid shortening (CURTIN and WOLEDGE, 1978; Homsher and Kean, 1978; Kodama and Yamada, 1979).

We thank Drs. R. C. Woledge, Nancy A. Curtin, and J. A. Rall for valuable advice in constructing the thermopile. We also thank Dr. T. Kobayashi for his help in constructing the thermopile. This work was supported in part by Grants-in-Aid for Scientific Research (548100, 56370006, 57222019) from the Ministry of Education, Science and Culture of Japan.

\section{REFERENCES}

Curtin, N. A. and Woledge, R. C. (1978) Energy changes and muscle contraction. Physiol. Rev., 58: 690-761.

Gordon, A. M., Huxley, A. F., and Julian, F. J. (1966) The variation in isometric tension with sarcomere length in vertebrate muscle fibres. J. Physiol. (Lond.), 184: 170-192.

HiLl, A. V. (1938) The heat of shortening and the dynamic constants of muscle. Proc. R. Soc. Lond. (Biol.), 126: 136-195.

HiLl, A. V. (1964) The effect of load on the heat of shortening of muscle. Proc. R. Soc. Lond. (Biol.), 159: 297-318.

Hill, A. V. (1965) Trails and Trials in Physiology, Arnold, London, pp. 304-330.

Hill, A. V. and Woledge, R. C. (1962) An examination of absolute values in myothermic measurements. J. Physiol. (Lond.), 163: 311-333.

Homsher, E., Irving, M., and Wallner, A. (1981) High-energy phosphate metabolism and energy liberation associated with shortening in frog skeletal muscle. J. Physiol. (Lond.), 321: $423-436$.

Homsher, E. and Kean, C. J. (1978) Skeletal muscle energetics and metabolism. Annu. Rev. Physiol., 40: 93-131.

Vol. 33, No. 6, 1983 
Homsher, E., Mommaerts, W. F. H. M., Richilutz, N. V., and Wallner, A. (1972) Activation heat, activation metabolism and tension-related heat in frog semitendinosus muscles. J. Physiol. (Lond.), 220: 601-625.

Homsher, E. and Rall, J. A. (1973) Energetics of shortening muscles in twitches and tetanic contractions. I. A reinvestigation of Hill's concept of the shortening heat. J. Gen. Physiol., 62: 663-676.

Huxley, A. F. (1957) Muscle structure and theories of contraction. Prog. Biophys. Biophys. Chem., 8: 255-318.

Huxley, A. F. (1980) Reflections on Muscle, Liverpool Univ. Press, Liverpool, 111 pp.

Irving, M., Homsher, E., and LebacQ, J. (1980) Dependence of the shortening heat on sarcomere length in frog skeletal muscle. Fed. Proc., 39: 1730.

IRving, M. and Woledge, R. C. (1981) The dependence on extent of shortening of the extra energy liberated by rapidly shortening frog skeletal muscle. J. Physiol. (Lond.), 321: 411422.

Irving, M., Woledge, R. C., and Yamada, K. (1979) The heat produced by frog muscle in a series of contractions with shortening. J. Physiol. (Lond.), 293: 103-118.

Jewell, B. R., Kretzschmar, K. M., and Woledge, R. C. (1967) Length and tension transducers. J. Physiol. (Lond.), 191 : 10 P-12 P.

KoDAma, T. and Y AMADA, K. (1979) An explanation of the shortening heat based on the enthalpy profile of the myosin ATPase reaction. In: Cross-bridge Mechanism in Muscle Contraction, ed. by Sugi, H. and Pollack, G. H. University of Tokyo Press, Tokyo, pp. 481 488.

Kushmerick, M. J., LARson, R. E., and Davies, R. E. (1969) The chemical energetics of muscle contraction. I. Activation heat, heat of shortening and ATP utilization for activation-relaxation processes. Proc. R. Soc. Lond. (Biol.), 174: 293-313.

LEBACQ, J. (1972) La chaleur de raccourcissement musculaire à différentes longueurs du sarcomère. J. Physiol. (Paris), 65: 440A.

MeYeR, K. H. and Haselbach, C. (1949) Rubber-like properties of hair keratin. Nature, 164: 33-34.

Rall, J. A., Homsher, E., Wallner, A., and Mommaerts, W. F. H. M. (1976) A temporal dissociation of energy liberation and high-energy phosphate splitting during shortening in frog skeletal muscles. J. Gen. Physiol., 68: 13-27.

Ricchiuti, N. V. and Mommaerts, W. F. H. M. (1965) Technique for myothermic measurements. Physiologist, 8: 259.

Yamada, K., Kometani, K., and Kobayashi, T. (1981) The relation between shortening heat and sarcomere length in frog skeletal muscle. J. Physiol. Soc. Jpn., 43: 373. 